\title{
Normal-Appearing White Matter Permeability Distinguishes Poor Cognitive Performance in Processing Speed and Working Memory
}

\author{
A. Eilaghi, A. Kassner, I. Sitartchouk, P.L. Francis, R. Jakubovic, A. Feinstein, and R.I. Aviv
}

\begin{abstract}
BACKGROUND AND PURPOSE: Secondary-progressive MS is characterized by reduced acute inflammation and contrast enhancement but with increased axonal degeneration and cognitive/clinical disability that worsens with advanced disease. Relative recirculation, extracted from DSC is a surrogate measure of BBB integrity. We hypothesized that normal-appearing white matter relative recirculation is reduced in cognitively impaired compared with nonimpaired secondary-progressive MS, reflecting more advanced disease.
\end{abstract}

MATERIALS AND METHODS: Cognitive performance was classified as impaired or nonimpaired by use of Minimal Assessment of Cognitive Function In MS test components. Demographic data, brain parenchymal fraction, WM lesion fraction, and weighted mean normal-appearing white matter relative recirculation were compared in cognitively dichotomized groups. Univariate and multivariate logistic regressions were used to study the association between cognitive test results and normal-appearing white matter relative recirculation.

RESULTS: The mean (SD) age of 36 patients with secondary-progressive MS studied was $55.9 \pm 9.3$ years; 13 of 36 ( $36 \%$ ) patients were male. A highly significant difference between normal-appearing white matter relative recirculation and WM lesion relative recirculation was present for all patients $(P<.001)$. Normal-appearing white matter relative recirculation in impaired patients was significantly lower than in nonimpaired subjects for the Symbol Digit Modalities Test $(P=.007)$, Controlled Word Association Test $(P=.008)$, and Paced Auditory Serial Addition Test $(P=.024)$. The Expanded Disability Status Scale demonstrated an inverse correlation with normal-appearing white matter relative recirculation $(r=-0.319, P=.075)$. After adjustment for confounders, significant normal-appearing white matter relative recirculation reduction persisted for the Symbol Digit Modalities Test $(P=.023)$ and the Paced Auditory Serial Addition Test $(P=.047)$ but not for the Controlled Word Association Test $(P=.13)$ in impaired patients.

CONCLUSIONS: Significant normal-appearing white matter relative recirculation reduction exists in cognitively impaired patients with secondary-progressive MS, localizing to the domains of processing speed and working memory.

ABBREVIATIONS: $\mathrm{rR}$ = relative recirculation; $\mathrm{NAWM}=$ normal-appearing white matter; $\mathrm{SPMS}=$ secondary-progressive MS; RRMS = relapsing-remitting MS

$\mathbf{M}_{\mathrm{o}}^{\mathrm{s}}$ $\mathrm{S}$ is the most common inflammatory demyelinating disease of the central nervous system. Although the underlying etiology of the disease is still largely unknown, inflammation, demy-

Received November 8, 2012; accepted after revision December 19.

From the Department of Medical Imaging (A.E., P.L.F., R.J., R.I.A.) and Department of Psychiatry (A.F.), Sunnybrook Health Sciences Centre, Toronto, Ontario, Canada; Department of Medical Biophysics (A.E.), University of Western Ontario, London, Ontario, Canada; Department of Medical Imaging (A.K., I.S., R.I.A.), University of Toronto, Toronto, Ontario, Canada; and Department of Physiology and Experimental Medicine (A.K.), Hospital for Sick Children, Toronto, Ontario, Canada.

Please address correspondence to Richard I. Aviv, MBChB, Sunnybrook Health Sciences Centre, 2075 Bayview Ave, Room AG 31, Toronto, Ontario M4N 3M5, Canada; e-mail: richard.aviv@sunnybrook.ca

三 Indicates article with supplemental on-line table

http://dx.doi.org/10.3174/ajnr.A3539 elination, and neurodegeneration are pathologically implicated and eventually cause long-term impairment. ${ }^{1}$ Cognitive impairment is reported in $40-70 \%$ of MS cases, ${ }^{2}$ and the frequency and expression of impairment increases with disease duration. ${ }^{3}$ Secondary-progressive MS is clinically distinct from relapsing-remitting MS. Patients with secondary-progressive MS do not manifest acute attacks ${ }^{4}$ and demonstrate diminished response to immunosuppressives, ${ }^{4}$ reduced lesional gadolinium enhancement, ${ }^{5}$ and diffuse NAWM inflammation and axonal injury. ${ }^{6}$

Traditional structural markers including WM lesion load and cortical and WM atrophy demonstrate variable correlation with cognitive impairment. ${ }^{7}$ Advanced MR imaging techniques are increasingly being investigated as surrogate markers of cognition. Techniques such as magnetization transfer imaging, ${ }^{8}$ diffusion- 
weighted imaging, ${ }^{9}$ functional MR imaging, ${ }^{10}$ MR spectroscopy, ${ }^{11}$ and perfusion imaging ${ }^{12}$ have previously been described. In RRMS, localized inflammation and BBB breakdown depicted with gadolinium-enhanced T1-weighted MR imaging are traditionally associated with disease activity. ${ }^{13,14}$ Unlike RRMS, SPMS is characterized by reduced acute inflammation and few contrastenhancing lesions. ${ }^{5}$ A number of mechanisms are described for the reduced apparent inflammation, including "compartmentalization" of the inflammatory response behind an intact $\mathrm{BBB},{ }^{6}$ balanced inflammation, activation of endogenous neuroprotection mechanisms, ${ }^{15,16}$ and continued neurodegeneration independent of inflammation. ${ }^{6,17}$ Despite a reduction of acute inflammatory changes, SPMS is associated with a higher prevalence of axonal degeneration, disability, and cognitive impairment, particularly in the domains of attention and processing speed. These functional domains may therefore serve as markers of more advanced axonal degeneration, distinguishing SPMS subgroups with more advanced disease. ${ }^{18}$

Relative recirculation ( $\mathrm{rR}$ ), a parameter extracted from DSC MR imaging, is shown to successfully identify regions of BBB breakdown in patients with brain tumors, ${ }^{19,20}$ Moyamoya syndrome, ${ }^{21}$ and acute ischemic stroke. ${ }^{22} \mathrm{rR}$ is a quantitative measure of contrast recirculation abnormalities after the first pass and is a practical surrogate for $\mathrm{BBB}$ permeability estimates that does not necessitate a separate dynamic contrast-enhanced MR imaging acquisition. ${ }^{22}$ The role of $\mathrm{rR}$ as a surrogate measure of $\mathrm{BBB}$ integrity in MS has not been evaluated. The purpose of this study was to compare rR in SPMS subgroups with and without cognitive impairment. We hypothesized that NAWM rR would be lower in cognitively impaired compared with nonimpaired patients with SPMS, reflecting a more advanced disease state. We also hypothesized that $\mathrm{rR}$ would be higher in WM lesions compared with NAWM.

\section{MATERIALS AND METHODS Patient Cohort}

The study was performed in accordance with our institution's guidelines for human research. Written informed consent was obtained for all imaging studies. Patients with clinically diagnosed SPMS were prospectively recruited from tertiary referral clinics. Two senior neurologists (20 years of experience) screened potential subjects to ensure they fulfilled the revised McDonald criteria. Exclusion criteria included patients with a history of drug/alcohol abuse, disease modifying drug or steroid use within the last 6 months, premorbid psychiatric history, head injury with loss of consciousness, concurrent medical diseases (eg, cerebrovascular disease), and MR imaging or contrast agent contraindications. Thirty-six patients with secondary-progressive MS fulfilled the screening criteria and were included in the study. Demographic data obtained included age, sex, years of education, and disease duration. Expanded Disability Status Scale assessment and cognitive testing was completed by a senior neurologist. No Expanded Disability Status Scale threshold was applied to patient eligibility. Neurologic examination, Expanded Disability Status Scale, and neurocognitive assessments were completed in order, on the same day within 6 hours of each other.

\section{Imaging Protocol}

Imaging parameters included volumetric T1 turbo field-echo (TR/TE/flip angle: $9.5 \mathrm{~ms} / 2.3 \mathrm{~ms} / 12^{\circ}$; number of averages: 1; FOV: $24 \mathrm{~cm}$; matrix size: $256 \times 164$; section thickness: $1.4 \mathrm{~mm}$ ); proton attenuation/T2 (TR/TE/flip angle: $2900 \mathrm{~ms} / 10.7 \mathrm{~ms} / 90^{\circ}$; FOV: $23 \mathrm{~cm}$; matrix: $256 \times 261$; section thickness: $3 \mathrm{~mm}$ ); fieldecho echo-planar imaging DSC (for rR calculation) (TR/TE/flip angle: $1610 \mathrm{~ms} / 30 \mathrm{~ms} / 60^{\circ}$; FOV: $22 \mathrm{~cm}$; section thickness: $4 \mathrm{~mm}$; matrix: $128 \times 128$; in-plane voxel size: $1.7 \times 1.7 \mathrm{~mm}$; no gap; signal bandwidth: $1260 \mathrm{~Hz} /$ pixel). Ten milliliters of gadobutrol (Gadovist; Bayer, Toronto, Canada) $(1 \mathrm{mmol} / \mathrm{mL})$ was administered by a power injector at a rate of $5 \mathrm{~mL} / \mathrm{s}$ followed by a $25-\mathrm{mL}$ bolus of saline at $5 \mathrm{~mL} / \mathrm{s}$. Sixty volumes were acquired at 1.6second intervals with the injection occurring at the 10th volume. DSC acquisition covered $10 \mathrm{~cm}$ ( 25 sections at $4 \mathrm{~mm}$ per section) and extended from the cerebral convexity to the midbrain level. Acquisition placement was supervised by an experienced neuroradiologist (8 years of experience) to ensure consistent coverage across patients.

\section{Image Analysis}

Gray matter, WM, and CSF were segmented from T1-weighted scans by use of a previously validated tissue segmentation method. ${ }^{23}$ Segmented regions were imported into Analyze 8.0 (Analyze AVW, Rochester, Minnesota), ${ }^{24}$ and WM lesion regions of interest were manually drawn. Brain parenchymal and WM lesion fractions were calculated as previously described. ${ }^{25}$ NAWM regions of interest were selected from within the WM tissue by use of four 16-voxel blocks located in anterior and posterior regions of both hemispheres. All WM lesion and NAWM regions of interest were subsequently co-registered to the equivalent DSC-MR imaging datasets by means of the rigid body algorithm of Statistical Parametric Mapping version 8 (SPM8; Wellcome Department of Imaging Neuroscience, London, United Kingdom). Contrast enhancement was systematically evaluated by use of a cross-reference tool by comparing FLAIR and proton attenuation/T2 lesions with precontrast and postcontrast imaging.

rR was calculated within each region of interest by use of in-house software (MR Analyst version 4.0) developed in Matlab (MathWorks, Natick, Massachusetts). ${ }^{22}$ In brief, selected DSC perfusion datasets were preprocessed by inspecting signal intensity versus time curves for outlier data points. Spurious points were identified by visual inspection and replaced with interpolated values. The first 2 dynamic phases were automatically omitted because magnetization cannot be assumed to have reached steady-state this early in the series. For each region of interest and each DSC image, the T2* relaxation rate was determined by evaluating the change in signal intensity from baseline and assuming ${ }^{26}$ :

$$
\Delta R_{2}{ }^{*} \text { measured }=-\ln (\Delta \mathrm{SI}) / \mathrm{TE}
$$

$\Delta R 2^{*}$ measured data were subsequently fitted to a $\gamma$-variate function to produce a theoretic first-pass $\Delta \mathrm{R} 2^{\star}$ theoretic curve, that is, one that was free of recirculation effects. The $\mathrm{rR}$ was calculated as described by Kassner et $\mathrm{al}^{27}$ as: 

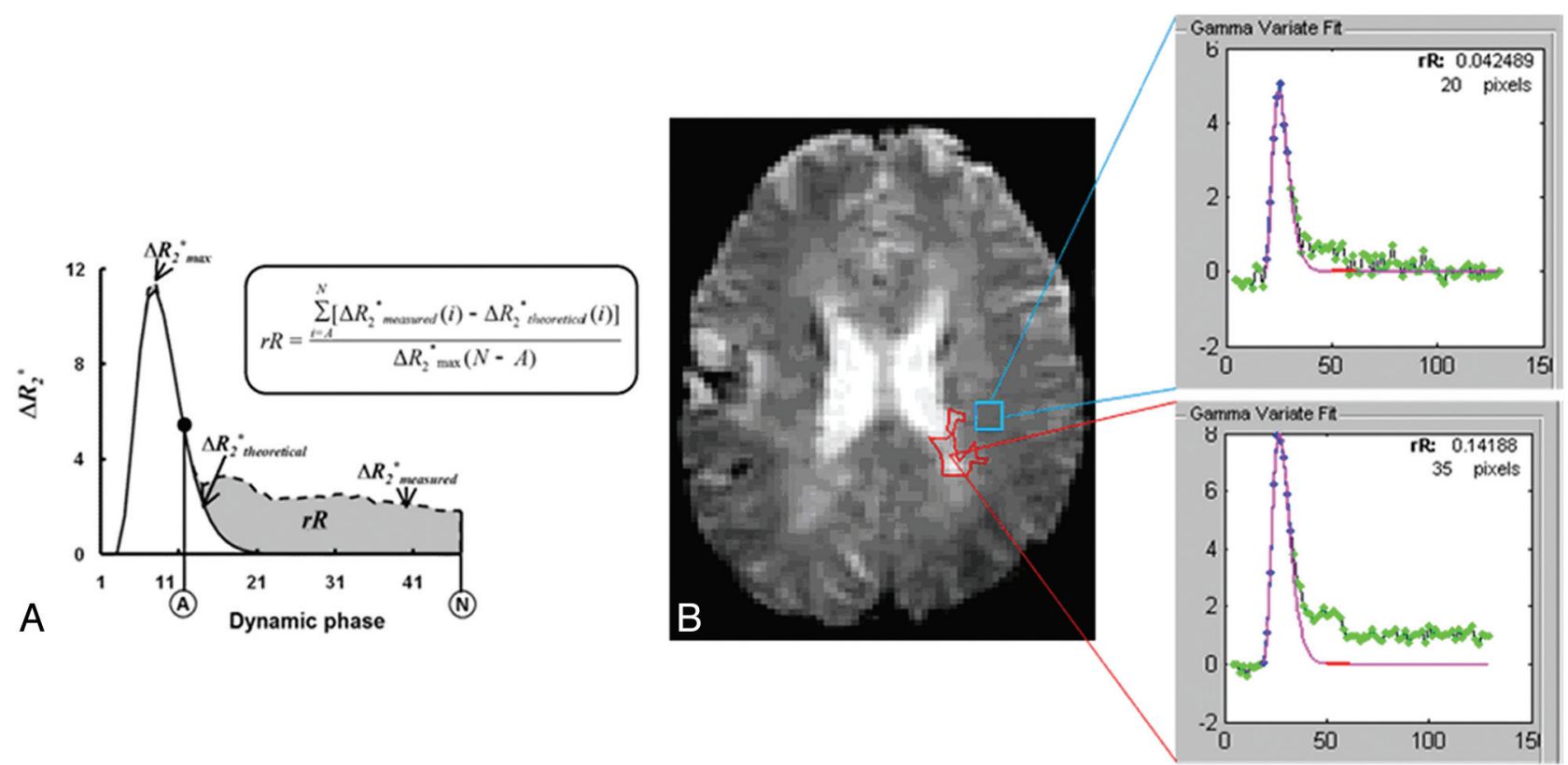

FIG 1. A, Estimation of $r R$ by use of the $\Delta R_{2}{ }^{*}$ versus time curve measured from the DSC regions of interest $\left(\Delta R_{2}{ }^{*}\right.$ measured $)$ as well as its $\gamma$-variate fit $\left(\Delta R_{2}{ }^{*}{ }{ }_{i t}\right)$. $\Delta R_{2}{ }^{*}$ max is the maximum of $\Delta R_{2}{ }^{*}$ theoretical. $A$ is the dynamic phase corresponding to the onset of the recirculation phase measured at half-height of the descending aspect of the $\Delta R_{2}{ }^{*}$ curve, and $N$ is the final DSC phase. $B$, Example of rR measurement: case 733, section 11, perfusion scan. MS lesion (red region of interest) and NAWM (blue region of interest) and rR calculation results. $r R$ for WM lesion is 0.142 ; rR for NAWM region is 0.042 .

2)

$$
r R=\frac{\sum_{i=A}^{N}\left[\Delta R_{2}{ }^{*} \text { measured }(i)-\Delta R_{2}{ }^{*}{ }_{\text {theoretical }}(i)\right]}{\Delta R_{2}{ }^{\star} \max _{\text {max }}(N-A)}
$$

where $\Delta R_{2}^{*}{ }_{\text {max }}$ is the maximum of $\Delta R_{2}{ }^{*}$ theoretical,$A$ is the dynamic phase corresponding to the onset of the recirculation phase measured at half-height of the descending aspect of the $\Delta R_{2}{ }^{*}$ curve, and $N$ is the final dynamic phase (Fig 1). Mean WM lesion rR and NAWM rR were determined for each patient by calculating the weighted average over WM lesion and NAWM regions of interest, respectively.

\section{Cognitive Assessment}

Cognitive assessments were performed by a neuropsychiatrist (20 years of experience) by use of an MS-specific neurocognitive tool, which is a 90-minute neurocognitive test proposed by an expert panel for clinical monitoring and research. ${ }^{28}$ The Minimal Assessment of Cognitive Function in Multiple Sclerosis is a comprehensive and efficient assessment tool consisting of 7 neuropsychological tests: Paced Auditory Serial Addition Test [working memory/ processing speed], Symbol Digit Modalities Test [processing speed], California Verbal Learning Test, 2nd edition [verbal memory], Brief Visuospatial Memory Test, revised [visuospatial memory], Delis-Kaplan Executive Function System [executive function], Controlled Word Association Test [verbal fluency], and judgment of line orientation [visuospatial perception].Impairment on an individual test was defined as scoring $>1.5$ standard deviations below normative data from healthy controls. Beck Depression Inventory and the Expanded Disability Status Scale were also obtained.

\section{Statistical Analysis}

Univariate comparisons of demographic data for impaired and nonimpaired groups were performed by use of the Mann-Whitney rank sum test. Demographic data were expressed as mean (SD) for continuous variables such as age, education, and disease duration; median with interquartile range for categorical variables such as the Beck Depression Inventory and the Expanded Disability Status Scale; and proportions for binary data such as sex. Segmented regional data for brain parenchymal fraction, gray matter fraction, WM fraction, and WM lesion fraction were similarly compared. The 10th and 90th percentile values for $\mathrm{rR}$ distribution of each group were calculated, and values beyond these limits were excluded. For each cognitive test, potentially confounding factors of age, sex, years of education, disease duration, Beck Depression Inventory score, Expanded Disability Status Scale, brain parenchymal fraction, WM fraction, WM lesion fraction, and gray matter fraction were compared. Potential confounders with $P$ values $<0.3$ (On-line Table) were included in further multivariate logistic regression. All statistical analyses were performed in SigmaStat3.5 (Systat Software, San Jose, California).

\section{RESULTS}

There were 36 patients, with a mean (SD) age of $55.9 \pm 9.3$ years; 13 of $36(36 \%)$ were male. Mean (SD) years of education and disease duration were 15.3 (2.6) and 20.4 (10.0). Median (IQR) for the Beck Depression Inventory and the Expanded Disability Status Scale were $8(5-11)$ and $6.5(6.0-7.0)$. Mean (SD) brain parenchymal fraction, WM fraction, WM lesion fraction, and gray matter fraction were 80.6 (4.1), 36.7 (3.0), 1.4 (1.2), and 41.5 (2.4), respectively. A highly significant difference between 


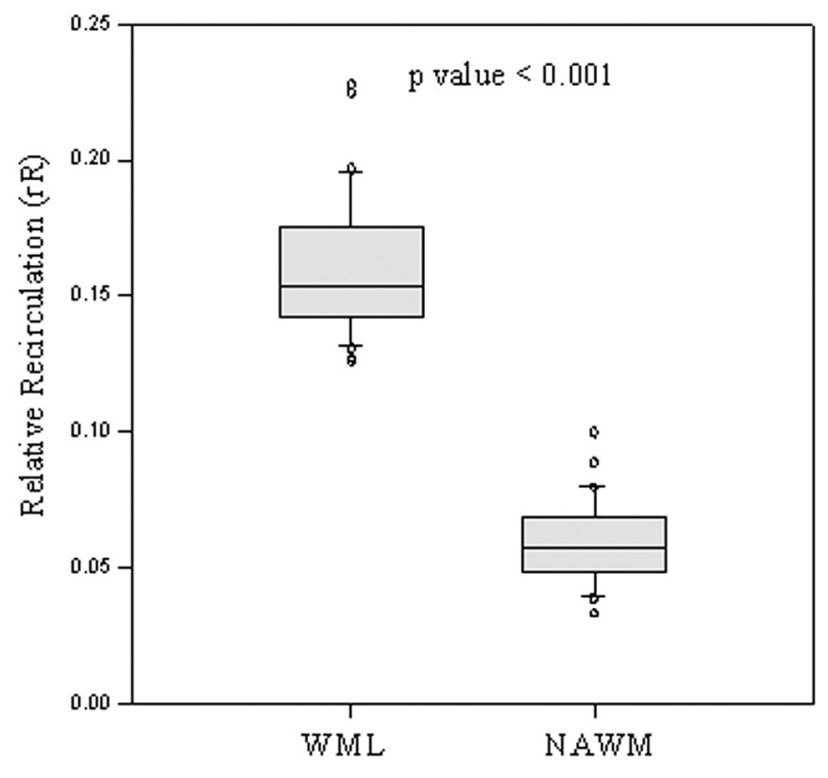

FIG 2. $r R$ values of NAWM and WM lesion.

Outcomes of multivariate logistic regression analyses for NAWM rR after controlling for potential confounders

\begin{tabular}{lcc}
\hline Cognitive Test & Odds Ratio $(5-95 \%$ CI) & $\boldsymbol{P}$ Value \\
\hline SDMT & $0.177(0.040-0.785)$ & .023 \\
COWAT & $0.662(0.002-2.226)$ & .130 \\
PASAT 1.6 & $0.053(0.003-0.961)$ & .047 \\
\hline
\end{tabular}

Note:-SDMT indicates Symbol Digit Modalities Test; COWAT, Controlled Word Association Test; PASAT, Paced Auditory Serial Addition Test.

Confounders for Symbol Digit Modalities Test: brain parenchymal fraction, WM fraction, WM lesion fraction; Controlled Word Association Test: WM fraction, WM lesion fraction, gray matter fraction; and Paced Auditory Serial Addition Test: brain parenchymal fraction, WM fraction, WM lesion fraction.

NAWM rR continues to be significantly lower in the impaired group for both the Symbol Digit Modalities Test and the Paced Auditory Serial Addition Test.

NAWM rR and WM lesion $\mathrm{rR}$ was present for all patients $(P<$ .001; Fig 2). No enhancing WM lesions were present in either SPMS subgroup.

No significant differences between NAWM rR in impaired and nonimpaired patients were observed for the Brief Visuospatial Memory Test $(P=.161)$, judgment of line orientation $(P=.493)$, California Verbal Learning Test $(P=.669)$, or Delis-Kaplan Executive Function System $(P=.680)$ impairment. NAWM rR was significantly lower in impaired patients than in nonimpaired subjects for the Symbol Digit Modalities Test $(P=.007)$, Controlled Word Association Test $(P=.008)$, and Paced Auditory Serial Addition Test $(P=.024)$ (On-line Table). The mean WM lesion fraction was higher in impaired patients (0.02) than in nonimpaired $(0.01)$ for all tests (Symbol Digit Modalities Test: $P=.020$; Controlled Word Association Test: $P=.014$; and Paced Auditory Serial Addition Test: $P=.033$ ). No significant correlation was found between NAWM $r$ with brain parenchymal fraction $(\rho=0.010 ; P=.955)$, WM fraction $(\rho=0.187 ; P=.272)$, WM lesion fraction $(\rho=$ $-0.260 ; P=.124)$, or GM fraction $(\rho=-0.295 ; P=.080)$.

After adjusting for confounders (Table 1), significant NAWM $\mathrm{rR}$ reduction persisted in impaired patients for the Symbol Digit Modalities Test $(P=.023)$ and Paced Auditory Serial Addition Test $(P=.047)$ (Table) but not for the Controlled Word Association Test impairment $(P=.13)$. Figure 3 illustrates the distribu- tion of rR values of NAWM for impaired and nonimpaired Symbol Digit Modalities Test and Paced Auditory Serial Addition Test groups. The Paced Auditory Serial Addition Test and Symbol Digit Modalities Test in impaired patients demonstrated a 3.2and 4.6-year longer disease duration than in their nonimpaired counterparts. The difference did not however reach clinical significance $(P=.3$ and $P=.6$, respectively).

The Expanded Disability Status Scale demonstrated an inverse correlation with NAWM rR (correlation coefficient $=-0.319$, $P=.075)$.

\section{DISCUSSION}

NAWM rR reduction was present in patients with secondaryprogressive MS with poor performance on the Paced Auditory Serial Addition Test and Symbol Digit Modalities Test representing deficits in processing speed and working memory. NAWM rR values were significantly lower in impaired patients persisting after correction for potential confounding factors. Secondarily, WM lesion $r R$ was higher than NAWM for all patients irrespective of cognitive impairment and in the absence of overt contrast enhancement.

Acute inflammation and BBB permeability in RRMS, manifesting as enhancing lesions, are linked with clinical and cognitive disability. The progressive phase of MS, however has a distinct disease pathogenesis compared with the acute or relapsing-remitting phases. ${ }^{29,30}$ SPMS is characterized by progressive axonal degeneration with clinical and cognitive disability but little lesion enhancement. A number of explanations for these findings are offered. Concepts of balanced inflammation, activation of endogenous neuroprotection mechanisms, ${ }^{15,16}$ entrapped inflammation behind intact or repaired $\mathrm{BBB},{ }^{6}$ and continued neurodegeneration independent of inflammation ${ }^{17}$ were previously proposed. Zeis et $\mathrm{al}^{15}$ demonstrated that genes known to be involved in anti-inflammatory and protective mechanisms are upregulated more regularly than pro-inflammatory mechanisms in NAWM in progressive MS. Correlation between brain atrophy and cognitive impairment increases with longer disease duration and SPMS subtype, in which impairment is reported in $50 \%$ of patients. ${ }^{2,3,18}$ In the present study, therefore, the association between cognitive impairment as a marker of disease severity and $\mathrm{rR}$ reduction as a surrogate marker of reduced measurable inflammation is expected. Supporting the association between disease duration, increased disability, and inflammation is the observed negative correlation between NAWM rR and disease severity (measured by the Expanded Disability Status Scale) in the present study. ${ }^{31}$ Additionally, cognitively impaired patients demonstrated longer disease duration than did cognitively nonimpaired patients, though this did not reach statistical significance.

Information processing speed and working memory are the most affected cognitive domains in patients with MS.,32 The Symbol Digit Modalities Test and Paced Auditory Serial Addition Test have been shown in large series to be sensitive indicators of impairments in these domains. ${ }^{33}$ Both the Symbol Digit Modalities Test and Paced Auditory Serial Addition Test correlate with several brain imaging findings such as lesion volume and central cerebral atrophy. ${ }^{34-36}$ The correlation expectedly increases with disease duration and SPMS subtype reflecting greater disease burden and axonal degeneration. ${ }^{18}$ Previous studies also show that 

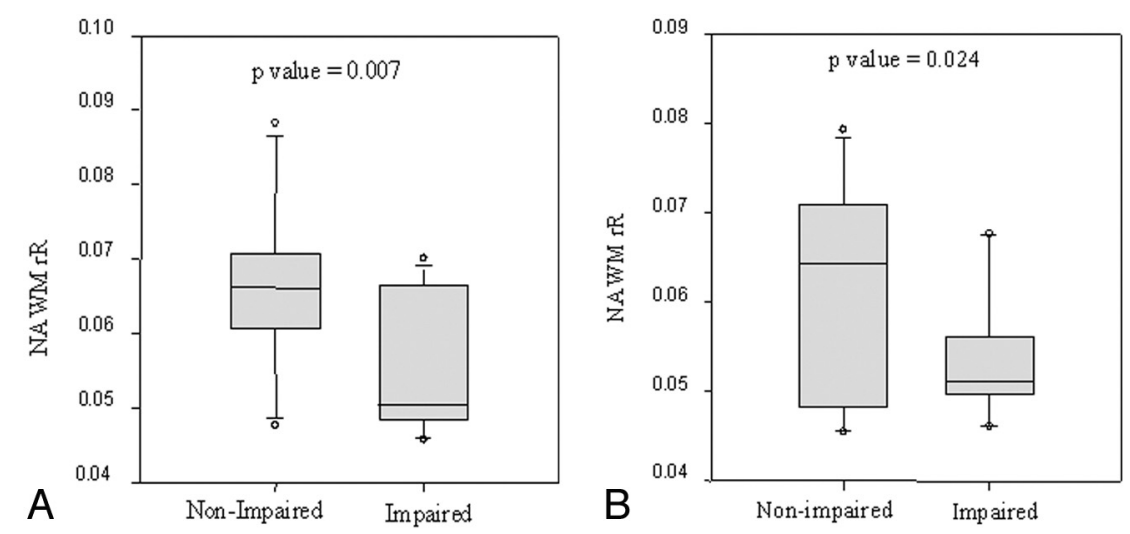

FIG 3. $r R$ values of NAWM are significantly lower in impaired than in nonimpaired groups by use of $A$, the Symbol Digit Modalities Test $(P=.007)$, and $B$, the Paced Auditory Serial Addition Test $(P=.024)$.

NAWM abnormalities, even independent of lesion location, may correlate with cognition response. ${ }^{37}$ NAWM abnormalities measured by diffusion-weighted MR imaging also predict the speed of information deficits in MS. ${ }^{3,14}$ Our study, like others, ${ }^{3}$ highlights the need for longitudinal study of associations between NAWM abnormalities and cognitive impairment.

In RRMS, contrast enhancement indicates increased risk of relapses and may also be associated with long-term dysfunction. ${ }^{38}$ However, contrast enhancement is weakly correlated with clinical attacks ${ }^{39}$ and is age-dependent. ${ }^{40}$ For enhancement to be present, a critical but unknown degree of BBB permeability must be present. Prior quantitative analysis of enhancement demonstrates the dose and time dependency of enhancement and the relative insensitivity of qualitative designation of enhancement. ${ }^{41} \mathrm{rR}$ as a surrogate marker of permeability was first studied in brain tumors ${ }^{19,20}$ and Moyamoya syndrome. ${ }^{21}$ More recently, rR was shown to strongly correlate $(r=0.67)$ with an established measure of BBB permeability (KPS) in acute ischemic stroke. ${ }^{22}$ Relative recirculation has the advantage of being extracted from standard DSC-MR imaging acquisitions without the need for a further contrast injection required for KPS determination. This is particularly important given the relationship between increasing contrast use and nephrogenic systemic fibrosis. ${ }^{42}$ Additionally, unlike KPS, the technique is model-free, with no need to obtain an input function avoiding a potential source of noise, ${ }^{43}$ as recently described. ${ }^{44}$ Last, $r R$ provides a surrogate measure of $\mathrm{BBB}$ permeability as a continuum as compared with the current "binary" view of contrast enhancement as present or absent.

The study is limited by a relatively small sample size. As an exploratory study, we analyzed a strictly homogeneous selection of patients with secondary-progressive MS, controlling for multiple potentially confounding structural parameters. Pragmatically, because of high lesion load, NAWM was selected from the bilateral centrum semiovale, representing the largest WM region available for interrogation. Cognition represents a complex interplay of multiple domains that cannot be captured by a single region of interest at any location. However, injury to the centrum is strongly associated with neurocognitive dysfunction in leukoaraiosis, ${ }^{45}$ carbon monoxide poisoning, ${ }^{46}$ and trauma, ${ }^{47}$ potentially mediated by "disconnection" of important projection and association fibers. ${ }^{45}$ However, despite these limitations, significant results were obtained for the Paced Auditory Serial Addition Test and the Symbol Digit Modalities Test, both sensitive indicators of information processing speed and working memory. Although perfusion and permeability techniques offer potential useful adjuncts to conventional imaging for monitoring disease activity, they are affected by recent bouts of acute inflammation, disease-modifying drugs, and steroid use. These limitations may limit their applicability in relapsing-remitting MS subgroups as the result of the heterogeneous nature of treatment and inflammation present but are less of a concern within the secondary-progressive MS subgroup. We limited our focus to patients with secondary-progressive MS because of the higher rate of cognitive impairment and relative stability of the disease. Future studies would benefit from control comparison and well-controlled relapsing-remitting MS cohorts. Nevertheless, the results appear clinically meaningful, warranting further exploration of the association between $\mathrm{rR}$, cognition, disease progression, and treatment response. If an association is found, $r R$ could be a useful target for future pharmacologic and rehabilitative treatment strategies.

\section{CONCLUSIONS}

Significant NAWM rR reduction exists in cognitively impaired patients with SPMS, localizing to the domains of processing speed and working memory.

Disclosures: Andrea Kassner—RELATED: Grant: Canada Research Chair, Comments: Salary award and research allowance. Anthony Feinstein-UNRELATED: Grants/ Grants Pending: MS Society of Canada;* Payment for Lectures (including service on speakers bureaus): Speakers honoraria paid to me from Merck Serono, Biogen, Teva, and Novartis; Royalties: Cambridge University Press. Richard Aviv—RELATED: Grant: MS Society, * PSI,* (*money paid to institution).

\section{REFERENCES}

1. Barnett MH, Sutton I. The pathology of multiple sclerosis: a paradigm shift. Curr Opin Neurol 2006;19:242-47

2. Chiaravalloti ND, DeLuca J. Cognitive impairment in multiple sclerosis. Lancet Neurol 2008;7:1139-51

3. Filippi M, Rocca MA, Benedict RH, et al. The contribution of MRI in assessing cognitive impairment in multiple sclerosis. Neurology 2010;75:2121-28

4. Compston A, Coles A. Multiple sclerosis. Lancet 2008;372:1502-17

5. Soon D, Tozer DJ, Altmann DR, et al. Quantification of subtle blood-brain barrier disruption in non-enhancing lesions in multiple sclerosis: a study of disease and lesion subtypes. Mult Scler 2007;13:884-94

6. Kutzelnigg A, Lucchinetti CF, Stadelmann C, et al. Cortical demyelination and diffuse white matter injury in multiple sclerosis. Brain 2005;128(Part 11):2705-12

7. Sperling RA, Guttmann CR, Hohol MJ, et al. Regional magnetic resonance imaging lesion burden and cognitive function in multiple sclerosis: a longitudinal study. Arch Neurol 2001;58:115-21

8. van Buchem MA, Grossman RI, Armstrong C, et al. Correlation of 
volumetric magnetization transfer imaging with clinical data in MS. Neurology 1998;50:1609-17

9. Benedict RH, Bruce J, Dwyer MG, et al. Diffusion-weighted imaging predicts cognitive impairment in multiple sclerosis. Mult Scler 2007; 13:722-30

10. Mainero C, Caramia F, Pozzilli C, et al. fMRI evidence of brain reorganization during attention and memory tasks in multiple sclerosis. Neuroimage 2004;21:858-67

11. Penny S, Khaleeli Z, Cipolotti L, et al. Early imaging predicts later cognitive impairment in primary progressive multiple sclerosis. Neurology 2010;74:545-52

12. Inglese $\mathrm{M}$, Adhya $\mathrm{S}$, Johnson $\mathrm{G}$, et al. Perfusion magnetic resonance imaging correlates of neuropsychological impairment in multiple sclerosis. J Cereb Blood Flow Metab 2008;28:164-71

13. Zivadinov R, Sepcic J. [Use of magnetic resonance imaging in the diagnosis and prognosis of multiple sclerosis]. Lijec Vjesn 2006;128:295-308

14. Filippi M, Agosta F. Imaging biomarkers in multiple sclerosis. $J$ Magn Reson Imaging 2010;31:770-88

15. Zeis T, Graumann U, Reynolds R, et al. Normal-appearing white matter in multiple sclerosis is in a subtle balance between inflammation and neuroprotection. Brain 2008;131(Part 1):288-303

16. Graumann U, Reynolds R, Steck AJ, et al. Molecular changes in normal appearing white matter in multiple sclerosis are characteristic of neuroprotective mechanisms against hypoxic insult. Brain Pathol 2003; $13: 554-73$

17. Lassmann H. Multiple sclerosis: is there neurodegeneration independent from inflammation? J Neurol Sci 2007;259:3-6

18. Lanz M, Hahn HK, Hildebrandt $\mathrm{H}$. Brain atrophy and cognitive impairment in multiple sclerosis: a review. J Neurol 2007;254(Suppl 2):II43-48

19. Jackson A, Kassner A, Zhu XP, et al. Reproducibility of T2* blood volume and vascular tortuosity maps in cerebral gliomas. J Magn Reson Imaging 2001;14:510-16

20. Jackson A, Kassner A, Annesley-Williams D, et al. Abnormalities in the recirculation phase of contrast agent bolus passage in cerebral gliomas: comparison with relative blood volume and tumor grade. AJNR Am J Neuroradiol 2002;23:7-14

21. Kassner A, Zhu XP, Li KL, et al. Neoangiogenesis in association with Moyamoya syndrome shown by estimation of relative recirculation based on dynamic contrast-enhanced MR images. AJNR Am J Neuroradiol 2003;24:810-18

22. Wu S, Thornhill RE, Chen $\mathrm{S}$, et al. Relative recirculation: a fast, model-free surrogate for the measurement of blood-brain barrier permeability and the prediction of hemorrhagic transformation in acute ischemic stroke. Invest Radiol 2009;44:662-68

23. Dade LA, Gao FQ, Kovacevic N, et al. Semiautomatic brain region extraction: a method of parcellating brain regions from structural magnetic resonance images. Neuroimage 2004;22:1492-502

24. Robb RA, Hanson DP, Karwoski RA, et al. Analyze: a comprehensive, operator-interactive software package for multidimensional medical image display and analysis. Comput Med Imaging Graph 1989;13:433-54

25. Aviv RI, Francis PL, Tenenbein R, et al. Decreased frontal lobe gray matter perfusion in cognitively impaired patients with secondaryprogressive multiple sclerosis detected by the bookend technique. AJNR Am J Neuroradiol 2012;33:1779-85

26. Rosen BR, Belliveau JW, Vevea JM, et al. Perfusion imaging with NMR contrast agents. Magn Reson Med 1990;14:249-65

27. Kassner A, Annesley DJ, Zhu XP, et al. Abnormalities of the contrast re-circulation phase in cerebral tumors demonstrated using dy- namic susceptibility contrast-enhanced imaging: a possible marker of vascular tortuosity. J Magn Reson Imaging 2000;11:103-13

28. Benedict RH, Fischer JS, Archibald CJ, et al. Minimal neuropsychological assessment of MS patients: a consensus approach. $\mathrm{Clin} \mathrm{Neu-}$ ropsychol 2002;16:381-97

29. Thompson AJ, Polman $\mathrm{CH}$, Miller $\mathrm{DH}$, et al. Primary progressive multiple sclerosis. Brain 1997;120(Part 6):1085-96

30. Heaton RK, Nelson LM, Thompson DS, et al. Neuropsychologica findings in relapsing-remitting and chronic-progressive multiple sclerosis. J Consult Clin Psychol 1985;53:103-10

31. Frischer JM, Bramow S, Dal-Bianco A, et al. The relation between inflammation and neurodegeneration in multiple sclerosis brains. Brain 2009;132(Part 5):1175-89

32. Benedict RH, Cookfair D, Gavett R, et al. Validity of the minimal assessment of cognitive function in multiple sclerosis (MACFIMS). J Int Neuropsychol Soc 2006;12:549-58

33. Drake AS, Weinstock-Guttman B, Morrow SA, et al. Psychometrics and normative data for the Multiple Sclerosis Functional Composite: replacing the PASAT with the Symbol Digit Modalities Test. Mult Scler 2010;16:228-37

34. Benedict RH, Weinstock-Guttman B, Fishman I, et al. Prediction of neuropsychological impairment in multiple sclerosis: comparison of conventional magnetic resonance imaging measures of atrophy and lesion burden. Arch Neurol 2004;61:226-30

35. Christodoulou C, Krupp LB, Liang Z, et al. Cognitive performance and MR markers of cerebral injury in cognitively impaired MS patients. Neurology 2003;60:1793-98

36. Lockwood AH, Linn RT, Szymanski H, et al. Mapping the neural systems that mediate the Paced Auditory Serial Addition Task (PASAT). J Int Neuropsychol Soc 2004;10:26-34

37. Dineen RA, Vilisaar J, Hlinka J, et al. Disconnection as a mechanism for cognitive dysfunction in multiple sclerosis. Brain 2009;132(Part 1):239-49

38. Zivadinov R, Bakshi R. Role of MRI in multiple sclerosis I: inflammation and lesions. Front Biosci 2004;9:665-83

39. Zivadinov R, Leist TP. Clinical-magnetic resonance imaging correlations in multiple sclerosis. J Neuroimaging 2005;15(4 Suppl):10S-21S.

40. Filippi M, Wolinsky JS, Sormani MP, et al. Enhancement frequency decreases with increasing age in relapsing-remitting multiple sclerosis. Neurology 2001;56:422-23

41. Silver NC, Tofts PS, Symms MR, et al. Quantitative contrast-enhanced magnetic resonance imaging to evaluate blood-brain barrier integrity in multiple sclerosis: a preliminary study. Mult Scler 2001;7:75-82

42. Rydahl C, Thomsen HS, Marckmann P. High prevalence of nephrogenic systemic fibrosis in chronic renal failure patients exposed to gadodiamide, a gadolinium-containing magnetic resonance contrast agent. Invest Radiol 2008;43:141-44

43. Cheng HL. Investigation and optimization of parameter accuracy in dynamic contrast-enhanced MRI. J Magn Reson Imaging 2008;28:736-43

44. Ingrisch M, Sourbron S, Morhard D, et al. Quantification of perfusion and permeability in multiple sclerosis: dynamic contrast-enhanced MRI in 3D at 3T. Invest Radiol 2012;47:252-58

45. O'Sullivan M, Morris RG, Huckstep B, et al. Diffusion tensor MRI correlates with executive dysfunction in patients with ischaemic leukoaraiosis. J Neurol Neurosurg Psychiatry 2004;75:441-47

46. Parkinson RB, Hopkins RO, Cleavinger HB, et al. White matter hyperintensities and neuropsychological outcome following carbon monoxide poisoning. Neurology 2002;58:1525-32

47. Kennedy MR, Wozniak JR, Muetzel RL, et al. White matter and neurocognitive changes in adults with chronic traumatic brain injury. J Int Neuropsychol Soc 2009;15:130-36 